\title{
Signal processing method of campus mutual aid platform based on mobile terminal
}

\author{
HUANG $\mathrm{Yi}^{1}, \mathrm{GAO} \mathrm{Jie}^{2}$ \\ \{jahg245@163.com¹, oijsfdiosd984@163.com²\} \\ (1.NanChang Business College of jxau,Gongqingcheng 332020,China; \\ 2.Modern Economics \& Management College of JXUFE, Gongqingcheng 332020,China)
}

\begin{abstract}
Intelligent terminal equipment is closely connected with wireless LAN, which makes the study and life of students and teaching staff more convenient, but its signal processing has a certain degree of lag. Based on this, the signal processing method of campus mutual aid platform based on mobile terminal is proposed. The channel is encoded and decoded so that the center frequency and noise of the signal can be corrected. After the timing and code optimization of the signal processing, the centralized processing of the signal can be realized. Experiments show that the method designed in this paper can guarantee high processing results and better stability under different degrees of interference.

Key word: Mobile terminal; Campus mutual aid platform; Signal processing method; Campus website; Educational administration management system
\end{abstract}

\section{Introduction}

With the rapid development of information technology, information technology has been integrated into all aspects of education. Educational informationization has become the main development direction of educational reform ${ }^{[1]}$. With the rapid development of information technology, "Internet of things", "cloud computing concept", "mobile smart campus" and other new things are gradually known by people, and affect the higher education environment and concept mode. In this profound change, the university digital campus has entered a new stage, towards the development of intelligent campus. On this basis, colleges and universities continue to research and develop digital campus service platform, such as the establishment of campus network, campus mutual platform, research office system and information management system. These systems and platforms can not only meet the daily office and teaching needs, but also facilitate the interaction between teachers and students. But there are also some problems: for example, in the early stage of construction, due to the lack of overall planning and guidance of the campus service platform, the poor connection between the school portal website, the provision of information services and OA, the utilization rate of information resources is low, and it is difficult for users to obtain the information and functions they want through a main page, not to mention the important information services and analysis and judgment Service. 
As one of the products of the development of information technology, intelligent terminal equipment is more popular and comprehensive. It is closely connected with wireless LAN, which makes the study and life of students and teaching staff more convenient. Intelligent terminal is gradually replacing desktops and laptops, and plays an important role in people's office, leisure and communication ${ }^{[2]}$. As the main user group of smart phones, college students have a certain knowledge base and are easy to accept new things. Because smart phones have become one of the main tools for communication between teachers and students, teachers and students can make full use of the fragmented time to acquire the knowledge they want at any time and anywhere, so they are not bound to sit in front of the classroom. At present, there are many colleges and universities in most parts of the country, each of which has its own campus portal. The functions of campus websites in Colleges and universities are relatively simple, mainly including the publicity of school running characteristics, professional introduction, teacher introduction, etc. The function of campus website in some colleges is more comprehensive, including data collection and educational administration management system. However, there is a lack of interaction between teachers and students, which can not meet the multi-faceted application needs of college students. Higher vocational college students need more in learning, entertainment, social and other aspects. Combined with the commonly used smart phones, a campus mutual aid platform suitable for the use of mobile terminals of higher vocational college students is designed, so that students can solve daily learning and life problems only by using mobile phones on campus. At the same time, the platform can also realize the interaction between students and teachers in their spare time. Teachers can manage students and release information. Students can also ask for leave from teachers in time, and can also query courses and scores independently, which makes teachers' teaching and students' knowledge learning more convenient and not limited by time and space.

\section{Signal processing method of campus mutual aid platform based on mobile terminal}

\subsection{Channel coding and decoding}

According to the traditional signal processing method, there are all kinds of interferences in the channel, which may lead to the distortion of the transmission information. In general, we use channel error correction coding to code baseband signal. In order to prevent signal distortion $^{[3]}$. Since the American scholars first proposed the reliable coding method of transmitting information in noisy channels in the early 20th century, error correction codes have been gradually developed and improved.

Channel coding technology improves error correction performance to some extent by adding redundant information bits to the transmitted information sequence. Through channel 
coding, when a certain instantaneous fading occurs in the channel, the transmitted data can still be recovered at the receiving end. At the beginning of baseband processing, channel coding transforms the original information sequence into a new code sequence containing more information bits by a specific algorithm. Then, the coded sequence is modulated so that it can be transmitted normally in the wireless channel.

In the digital communication system, the methods of information error control include feedback retransmission and forward error correction coding. In the feedback retransmission mode, the error code will be fed back, and then the transmitter will retransmit. Forward error correction code has no feedback mechanism, but encodes the error correcting information bits to the information sequence to be sent. The receiver receives the transmitted data, and the received redundant bits can find the error and achieve the purpose of error correction at the receiver. So this kind of forward error correction code reduces the efficiency of the channel by adding redundant bits, but it can make a lot of error codes can be correctly recovered at the receiver. When the forward error correction code is not used, the transmitter that generates the error code must resend the information bits. If the efficiency loss caused by the increase of redundant bits is less than the loss caused by the retransmission of error codes, the forward error correction code actually improves the efficiency of the channel. One of the most important parameters of FEC code is the code rate, which describes the ratio of loaded input bits to output bits with a decimal. For example, for a code rate of $1 / 2$, two information bits are output for each input of one information bit, and one bit of redundant bit information is added.

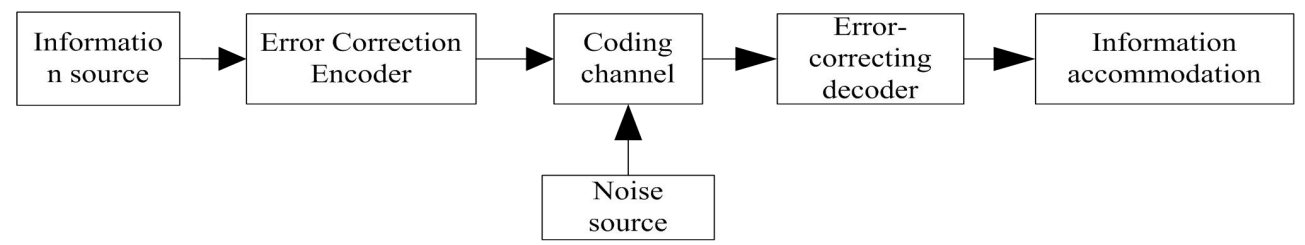

Fig. 1 forward error correction coding communication

Like the previous $802.11 \mathrm{a} / \mathrm{g}$, in $802.11 \mathrm{n}$, the forward error correction code continues to use convolutional coding. Convolutional code has high speed and simple hardware structure ${ }^{[4]}$. In this paper, the parameters of convolutional coding are $(2,1,6)$. This parameter is based on an example of $802.11 \mathrm{n}$. The selection of convolutional coding parameters in $802.11 \mathrm{n}$ is based on the experimental results considering the factors such as bit error rate and transmission rate. After coding, the transmitter needs to decode at the receiver. At present, viterbi decoding is most commonly used. Viterbi decoding is a decoding method based on "maximum similarity". By reproducing the path of information bits on the grid during convolutional coding, the most likely path is found as the output of decoding. When the constraint length is not large, the 
advantages of Viterbi decoding are particularly obvious. The hardware structure is relatively simple and the speed is relatively fast. Through the optimization of some key modules, a Viterbi decoder with high performance is designed to facilitate the real-time processing of signal data later.

\subsection{Adjustment of center frequency}

To filter signals with different frequencies, a filter with different center frequencies is needed, and the center frequency of the wavelet filter is related to the sampling frequency and the scale of the wavelet. At the same time, signal frequency and sampling frequency are closely related, that is, different sampling frequency is required for different frequency signals. If the sampling frequency is selected too high, there will be too many sampling points in a signal cycle, resulting in oversaturation. However, if the sampling frequency is too low, it may not satisfy the sampling theorem and can not accurately reflect the signal components, resulting in the decrease of measurement accuracy. On the premise of accurately reproducing the measured signal, we can adjust the center frequency of the filter through the following three paths to realize the filtering of different frequency signals.

First of all, changing the series of wavelet decomposition, that is, changing the scale to move the passband of the filter on the frequency axis, so that the signal components of different frequency bands pass through, which is very good for the signal with little frequency change.

Secondly, the data is extracted at intervals. To adapt to the change of signal frequency, the method of extracting data from each other is used. Sampling data every certain number of points, which is equivalent to reducing the sampling frequency. For the extracted data, wavelet transform of different series is still used to measure the value at lower frequency.

Finally, change the sampling frequency. In practical application, the data extraction method based on the number of sampling points is limited. The center frequency of wavelet filter is adjusted by directly changing the actual sampling frequency to meet the requirements of different frequency signals. As long as the sampling frequency is set correctly, the measurement of signal frequency is quite accurate. By changing the sampling frequency, very low frequency band (below $1 \mathrm{~Hz}$ ) and very high frequency band (above $2000 \mathrm{~Hz}$ ) can be measured. Through a large number of practices, it is found that the sampling frequency is set to 20 times of the signal frequency, and the accurate frequency measurement results can be obtained by calculating the period of the data after decomposing to level 4 wavelet without sampling points.

It should be noted that the effective adjustment of the center frequency is for the follow-up signal noise reduction processing, so that the campus mutual aid platform can play a 
better role.

\subsection{Signal noise correction}

In the process of signal acquisition, transmission and storage, it is often interfered and influenced by various kinds of noise, which leads to signal degradation. Moreover, the quality of signal preprocessing algorithm is directly related to the effect of subsequent signal processing, such as signal segmentation, target recognition, edge extraction, etc. In order to obtain high-quality digital signal, it is necessary to reduce the noise of the signal, keep the integrity of the original information as much as possible (i.e. the main features), and remove the useless information in the signal. Therefore, noise reduction has always been the focus of signal processing and computer vision research.

The ultimate goal of signal video denoising is to improve the given signal and solve the problem of signal quality degradation caused by noise interference. The denoising technology can effectively improve the signal quality, increase the signal-to-noise ratio, and better reflect the information of the original signal. Among the existing de-noising algorithms, some of them have achieved good results in low-dimensional signal processing, but they are not suitable for high-dimensional signal processing; or they have good de-noising effect, but they lose part of the signal edge information. At the same time, some researches are devoted to studying the edge information of the detected signal and retaining the signal details ${ }^{[5]}$. How to find a better balance between resisting noise and keeping details has become the focus of this paper.

Wavelet shrinkage method is the most widely used method at present. There are two kinds of wavelet shrinkage method: the first one is threshold shrinkage. Because threshold shrinkage is mainly based on the following facts, that is, the larger wavelet coefficients are generally based on the actual signals, while the smaller ones are largely noise. Therefore, by setting a suitable threshold value, the coefficients smaller than the threshold value can be set to zero, while the wavelet coefficients larger than the threshold value can be retained. Then the estimated coefficients are obtained by the threshold function mapping, and finally the estimated coefficients are inversely transformed to achieve denoising and reconstruction ${ }^{[6]}$. The second type is proportional shrinkage, which is called proportional shrinkage by judging the degree of noise pollution of coefficients and introducing various measurement methods (such as probability and membership) to determine the proportion of shrinkage. The research of signal processing method based on PDE is also a hot research direction of signal denoising, and has made some achievements in theory and practical application. Its denoising process is to establish the initial condition that the noise signal is a certain nonlinear PDE, and then solve the PDE to get the solution at different times, that is, the filtering result. Perona and Malik 
proposed a PDE based nonlinear diffusion filtering method (hereinafter referred to as P-M). The anisotropic denoising model determines the diffusion speed according to the gradient value of the signal, so that it can take into account the requirements of noise elimination and edge preservation. These methods, represented by P-M model, have been widely used in signal enhancement, signal segmentation and edge detection, and have achieved good results. P-M is a non-linear anisotropic method, which aims to overcome the shortcomings of fuzzy edge and edge position moving in linear filtering method. Basic idea: reduce the diffusion coefficient where the signal feature is strong, and enhance the diffusion coefficient where the signal feature is weak. Although the p-m equation has achieved some results in suppressing noise and retaining important features of signal, it is ill conditioned and unstable. CATT et al. Improved the equation by convoluting the Gaussian kernel with the signal and then using its gradient modulus to estimate the edge information of the signal. The optimized symmetric exponential filter is used to smooth the signal, and then the gradient modulus is used to estimate the edge information of the signal. The basic idea of these two estimation methods is to reduce the interference of noise, extract the edge feature information of signal more truly, so as to control the diffusion behavior of P-M equation better with the edge information. TV method is proposed by Rudin Osher and Fatemi. Based on the idea of variational method, it determines the energy function of signal, and achieves the purpose of smooth denoising by minimizing the energy function of signal. It is now a popular signal restoration method.

In this paper, the noise reduction algorithm of P-M equation and TV method is mainly considered. In the new algorithm, not only the important features of noise suppression and signal retention are studied, but also the minimization of signal energy function to achieve smooth noise reduction is studied. According to the characteristics of mobile intelligent terminal camera, such as uneven, small size and so on, the denoising algorithm is studied. For many low and medium pixel cameras, after denoising, the camera yield can be improved, which conforms to the goal and direction of high performance and low cost.

\subsection{Signal processing timing and code optimization}

The processing timing of each module is arranged in our method. The signal is processed in two channels (high frequency band channel and low frequency band channel). Each frame of the low frequency band channel is equivalent to the $\mathrm{K}$ frame of the high frequency band signal in the time span ${ }^{[7]}$. When the high-frequency band signal has enough $\mathrm{K}$ frames, one frame of low-frequency band signal processing can be carried out. If all the calculations are completed in the next high-frequency band signal frame, the calculation is too centralized. In order to enable the algorithm to handle higher speed control, it is necessary to arrange the processing time sequence of each module of the algorithm appropriately, so as to spread the 
calculation as far as possible, so as not to occur the situation that DSP is too late to handle. The FFT of the low-frequency band signal can only be processed when all the points of the frame are complete, so it can not be distributed to the intra processing of the high-frequency band signal, so the corresponding transfer function estimation, IFFT and other processing related to the output of the next frame before interpolation need to be completed in a high-frequency band signal frame. However, the decimation filtering with a large amount of computation can be distributed in each high-frequency band signal frame. When a high-frequency band signal frame data is ready, the corresponding decimation filtered signal can be calculated. The interpolation filtering of the output signal can also be distributed to each high-frequency band signal frame to complete. Each time the output stack is needed, the interpolation filtering is carried out to calculate the corresponding The output of the low-frequency band and the output of the high-frequency band are superposed. Therefore, the frame with the largest amount of calculation occurs when the data of the low-frequency band signal frame is ready. In this high-frequency band signal frame, it is necessary to extract the corresponding signal, estimate the spectrum of the low-frequency band input and output signal, estimate the transfer function and solve the output signal with positive $\mathrm{ft} .1$ service frame low-frequency band signal output interpolation filtering. Every k high-frequency band signal frames, there will be a large amount of computation. Under this arrangement and after the code manual optimization mentioned immediately, the control algorithm can complete the control of 8-channel input and 1-channel output with a control frequency of up to 9.skhz. The original single resolution control algorithm can only realize the optimization of 9.skhz control algorithm in the system of 8-Input and 1-output, which in fact includes the module calculation timing problem mentioned above. In addition, for each module, the optimization process mainly includes two steps: first, the optimization of $\mathrm{C}$ program before assembly; second, the optimization of assembly code. This is a good idea of algorithm optimization. High level language is relatively easy to write, which can make some optimization work that computers can do, and then optimize the code that some computers can't achieve efficient optimization manually.

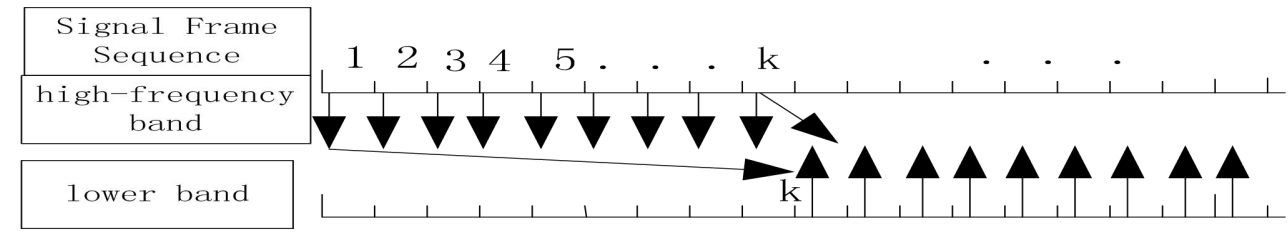

Fig. 2 Calculation timing of each module

In the whole implementation process of the method, the modules that account for a large 
amount of calculation include: decimation filtering module, FFT, IFFT and interpolation filtering module. The cores of FFT and IFFT are provided by TI company and have been optimized manually. Therefore, only decimation filtering module and interpolation filtering module are needed to be optimized. Considering that the decimation filtering in the control algorithm is carried out by frame and does not require continuous output, the direct filtering method can be used. After filtering, one k-1 signal in each k-point will be discarded and only one point will be reserved. Therefore, in the filtering calculation, we only need to calculate the point that needs to be reserved. In this way, the 160 order decimation filter is actually equivalent to a 160 order ordinary filter, so the calculation is not too large. In the filtering of each frame data, there are two sources of original data: current frame data and part of historical data.

The filtering process is composed of two layers of loops, the outer loop is the number of output points, and the inner loop completes the product of the filter sequence and the corresponding data, and sums it. The number of instructions in the inner loop determines the amount of calculation. If we need to judge whether the original data of convolution operation comes from the current frame or the historical data in the inner loop, then we know that at least seven or eight instructions are needed to make such a judgment in assembly code, and the calculation amount will increase greatly. Therefore, before filtering, it is necessary to combine the current frame data and historical data into a group. The DSP's on-chip RAM supports dualaccess, that is, each instruction cycle can be accessed twice, while the filtering operation accesses the data very frequently, so it is better to copy the original data and filter coefficients into the on-chip RAM before filtering. If the on-chip RAM is not large enough, then at least copy the filter coefficients into the on-chip, which is good for increasing the filtering speed. The next step is to use the assembler and optimizer provided by TI company for preliminary optimization to get the assembly code. On this basis, artificial optimization is carried out. The focus of artificial optimization is to reduce the number of instructions in the inner loop. The result of optimizer optimization is that the inner loop contains three instructions. In fact, C3x and $\mathrm{c} 4 \mathrm{x}$ series DSP contain a multiplier and an adder, which can handle one multiplication and one addition at the same time. Therefore, the optimal result is that the inner loop should contain only one instruction, so the effect of artificial optimization is very obvious. The optimization process of interpolating filter is basically the same, and it also goes through two processes: $\mathrm{C}$ language optimization and assembly code optimization. Table 1 and table 2 show the changes of calculation amount in the optimization process of decimation filter and interpolation filter respectively. Note that the data given here is the calculation amount used in the whole algorithm, including FFT and IFFT, because it is difficult to directly measure the 
calculation amount of a single module through experiments. In the experiment, the control mode is 8 inputs ( 1 control channel and 7 monitoring channels), 1 output, the sampling frequency is $12 \mathrm{khz}$, the maximum control frequency is $4680 \mathrm{hz}$, and the data per frame is 1024 points.

Table 1 change of calculation amount of control algorithm in the process of decimation filter optimization

\begin{tabular}{|c|c|}
\hline Optimizing content & $\begin{array}{l}\text { Calculations for the whole control } \\
\text { algorithm }\end{array}$ \\
\hline Before Optimization & 0.9 \\
\hline $\begin{array}{l}\text { Merge historical data with current frame } \\
\text { data }\end{array}$ & 0.83 \\
\hline $\begin{array}{l}\text { Filter coefficient copied to on-chip } \\
\text { RAM }\end{array}$ & 0.805 \\
\hline $\begin{array}{l}\text { The original data is copied to the in - } \\
\text { chip RAM. }\end{array}$ & 0.793 \\
\hline Assembly code optimization & 0.709 \\
\hline
\end{tabular}

Table 2 change of calculation amount of control algorithm in the process of interpolating filter optimization

\begin{tabular}{cc}
\hline Optimizing content & $\begin{array}{c}\text { Calculations for the whole control } \\
\text { algorithm }\end{array}$ \\
\hline $\begin{array}{c}\text { Before Optimization } \\
\text { Merge historical data with current frame } \\
\text { data }\end{array}$ & 0.709 \\
Filter coefficient copied to on-chip RAM \\
The original data is copied to the in - \\
chip RAM. & 0.67 \\
Assembly code optimization & 0.57 \\
\hline
\end{tabular}

Through the optimized algorithm, the highest frequency of 9.skhz can be controlled (other conditions remain unchanged, such as 8 input, 1 output, 1024 points per frame, etc.), at this time, the sampling frequency is $24 \mathrm{khz}$, and the calculation amount reaches 0.9944 . It can be seen that our filter design enables the system to achieve the best performance. Table 5.6 shows the calculation amount comparison between the original single resolution control algorithm and the new multi-resolution control algorithm under the control of different highest control frequency, 1 input control channel, 7 monitoring channels and 1024 points frame, so as 
to facilitate the subsequent centralized processing of signals.

\subsection{Centralized signal processing}

After the completion of the above several links, the final centralized signal processing is mainly realized by the empirical mode method. Empirical mode decomposition method can decompose non-stationary and non-linear signals into a set of stable and linear sequences, i.e. eigenmode function ${ }^{[8]}$. According to Huang's definition, the IMF of each stage should meet two conditions:

(1)The extreme point and zero crossing point of data appear alternately, and the number is equal or at most different at any point;

(2)At any point, the mean value of envelope defined by local maximum and local minimum must be zero. The filtering algorithm is as follows:

A. For input signal $x(t)$, determine all extreme points of $x(t)$.

B. The upper and lower envelope lines of $x(t)$ are obtained by fitting the maximum and minimum points with cubic spline function.

C. Subtract the mean value of the upper and lower envelopes from the original data series.

Average curve:

$$
m(t)=\left[e_{\min }(t)+e_{\max }(t)\right] / 2
$$

$m(t)$ represents the average curve, $e_{\min }(t)$ and $e_{\max }(t)$ represent the minimum and maximum value of the input signal respectively.

Detail signal:

$$
s(t)=x(t)-m(t)
$$

$s(t)$ stands for detail signal.

D. Generally, $s(t)$ does not meet the conditions of IMF, so the above steps need to be repeated for iterative processing ${ }^{[9-10]}$, and the iterative stop criteria given are:

$$
S D=\sum_{t=0}^{T}\left|S_{n-1}(t)-S_{n}(t)\right|^{2} / \sum_{t=0}^{T} S_{n-1}^{2}(t)
$$

SD is the screening threshold value, which is generally $02-0.3$. If SD is less than this threshold value, the filtering iteration will end. After the iteration meeting the stop criteria, the $s(t)$ is the effective IMF, and the remaining signals enter the next screening process. After several times of screening, the original data sequence is decomposed into a group of IMF components and a residual, and the IMF obtained is stable. The results obtained by Hilbert 
transform can well analyze non-linear and non-stationary signals.

\section{Experiment test and analysis}

In order to verify the improvement of the signal processing method of the design platform, a simple and intuitive test experiment is designed by using the GUI tool of MATLAB. The function of this system is to decompose the input signal with traditional EMD and EEMD, to display the IMF components and instantaneous frequency of each modal function after signal decomposition, and to describe the Hilbert time spectrum.

\subsection{Parameter setting}

The variance of white noise and the number of noise groups (range 1-500) can be set freely on the parameters. When the variance is set to 0 and the number of noise groups is selected to 1 , the system realizes the function of traditional EMD decomposition. The EEMD decomposition function adds the above set white noise to the signal and depicts the Hilbert time spectrum of the input signal. Display IMFs function can display IMF components and instantaneous frequency after signal decomposition in the form of pop-up Fig.

\subsection{Test results and analysis}

The specific test results are as follows:

Firstly, the multi-component ideal sample signal is decomposed, and the signal structure is as follows:

$$
x(t)=\sin \left(2 \pi f_{1} t\right)+\sin \left(2 \pi f_{2} t\right)+\sin \left(2 \pi f_{3} t\right)+\sin \left(2 \pi f_{4} t\right)
$$

The normalized frequency is: $f_{1}=0.02, f_{2}=0.05, f_{3}=0.1, f_{4}=0.2$.

EMD decomposition method should decompose the signal containing four frequency components into four IMF components containing single frequency information. The decomposition results are shown in Figure 3.

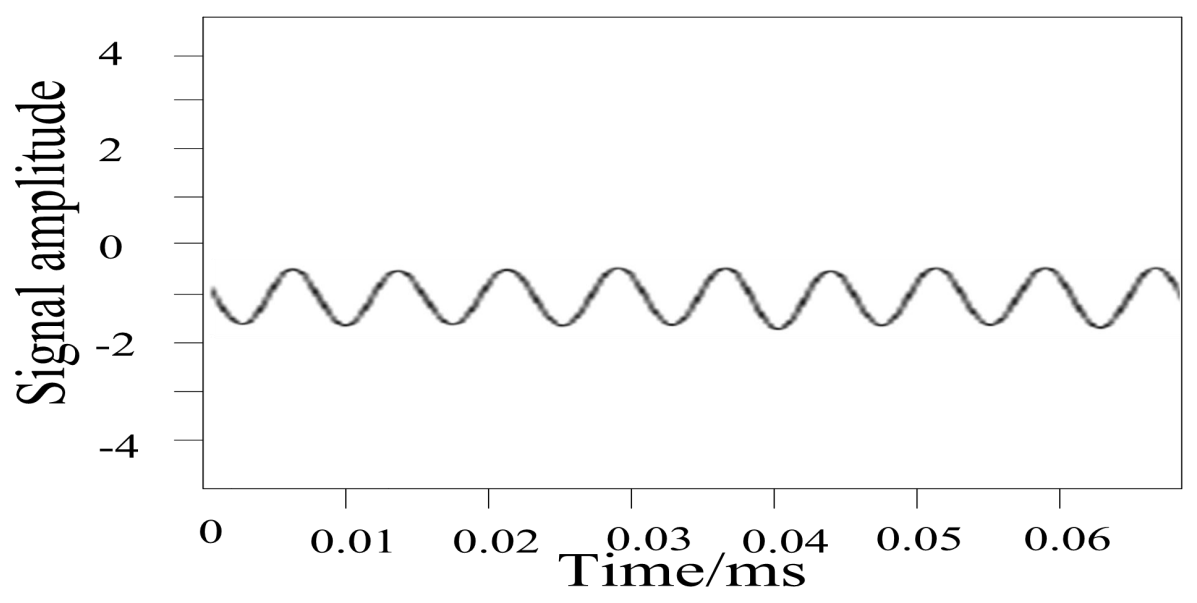


Fig. 3 Hilbert spectrum of traditional EMD for ideal signal

It can be seen that the traditional EMD decomposition method has a very good effect for the ideal signal without interference. The four frequency components are clearly displayed on the Hilbert spectrum. A group of actual signals with interruption interference are decomposed, and the results are shown in Fig. 4 and Fig. 5.

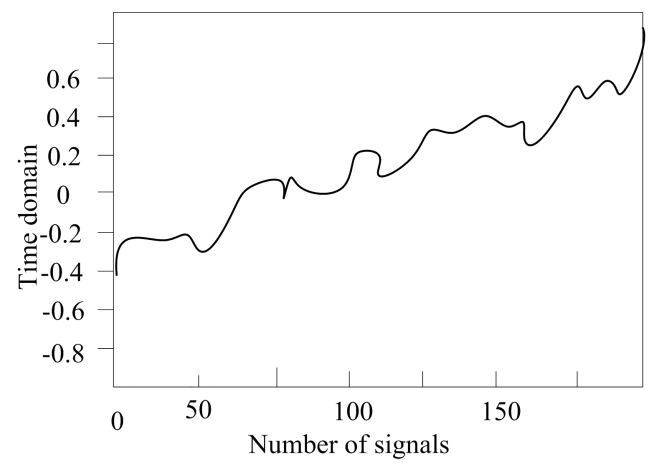

Fig. 4 Time domain diagram of actual signal

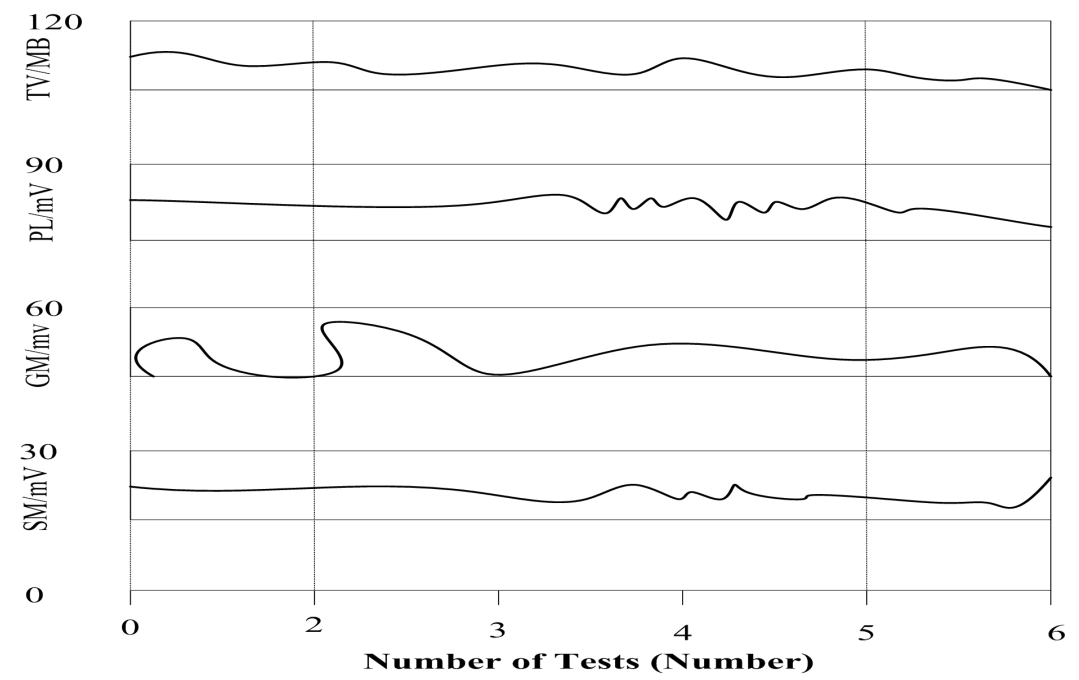

Fig. 5 EEMD decomposition of signal

It can be seen from the spectrum that the low frequency components are mixed together and difficult to distinguish.

After analyzing the EEMD processing method, 100 groups of Gaussian white noise with 
standard deviation of 0.2 are added, and the results are shown in Figure 4. Through the comparison of Hilbert spectrum, it can be seen that the processing results have been greatly improved.

\section{Conclusions}

Through the research and update of the signal processing method of campus mutual aid platform of mobile terminal, the interaction between university students and teachers in their spare time is realized, which provides convenience for teachers' classroom management. At the same time, students can also conveniently ask teachers for leave. Students can check their course scores here. Teachers and students can also understand the important information of campus through the platform. In this paper, the signal processing method of campus mutual aid platform based on mobile terminal is designed. By comparing with the traditional signal processing method, it is proved that the method designed in this paper can guarantee higher processing results and better stability under different degrees of interference.

\section{Fund projects}

Science and technology project of Jiangxi Provincial Department of education in 2019, Poject name:Research and implementation of intelligent delivery terminal based on mobile Internet and AI (GJJ191579)

\section{Reference}

[1] Xiao Lu, Wang Yihong, Chen Bo, et al. Analysis and Research on Functional Requirements of Campus Mobile Applications [J]. Computer Knowledge and Technology,2018,14(2):280-282.

[2] Chen Shilei The practice of hybrid learning model based on mobile terminal in e-commerce specialty[J]. Computer knowledge and technology,2018,14 (1):282-284.

[3] Yang Ying. Design and Research of Campus Human Environment Platform Based on Mobile Intelligent Terminal in Big Data Era [J]. Journal of Jilin Institute of Education,2018,34(4):154-156.

[4] Wei Yandi. Application of Digital Signal Processing Technology in Electronic Information Engineering [J]. Electronic Technology and Software Engineering,2019,21(8):111-113.

[5] Sun Fat Liu, Zenglu Li. Research and Practice on the Construction of "Mobile Intelligent Campus" in Local Undergraduate Colleges: A Case Study of Sanming College [J]. Journal of Taiyuan University (Natural Science Edition),2018,36(2):52-55.

[6] Geng Yanxiang, Pu Yanhong, Chen Yu, et al. Design of Virtual Experimental Platform for Digital Signal Processing [J]. Education and Teaching Forum,2018,45(36):274-276.

[7] Cai Zhongxing, Chen Peihang. Research and Realization of Smart Campus One - Stop Service Platform Based on Mobile Terminal [J]. Journal of China Network Institute of Technology,2018,21(5):68-71.

[8] Fan Xiaochun, Lu Yong, Tao Liang. Research on the Construction of Intelligent Campus in 
Universities in Mobile Internet Environment [J]. Journal of Jinling Institute of Science and Technology,2017,33(01):52-55.

[9] Luo Jinling. Research and Realization of Smart Campus One - Stop Service Platform Based on Mobile Terminal [J]. Computer Knowledge and Technology,2017,13(28):36-37 47.

[10]xinyan hu, wenxiao ho, xiaoyan che, et al. Design of Experimental Simulation Platform for Digital Signal Processing Based on MATLAB GUI [J]. Electronic Technology and Software Engineering,2019, 12(08):58-59. 